The ABCR score was validated and proved to perform better than the ART score in distinguishing between patients prognoses

(IDDF2021-ABS-0042 Figure 1. Overall survival of ABCR 3 groups,

IDDF2021-ABS-0042 Figure 2. Overall survival of ABCR 3 groups,

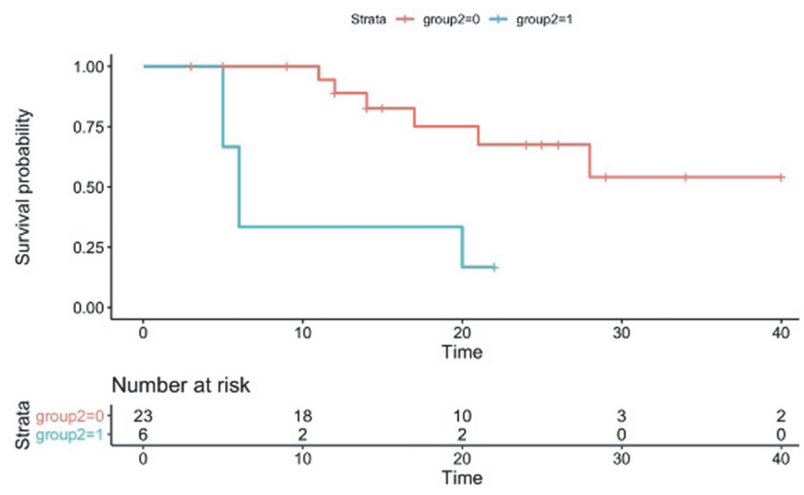

Abstract IDDF2021-ABS-0042 Figure 2

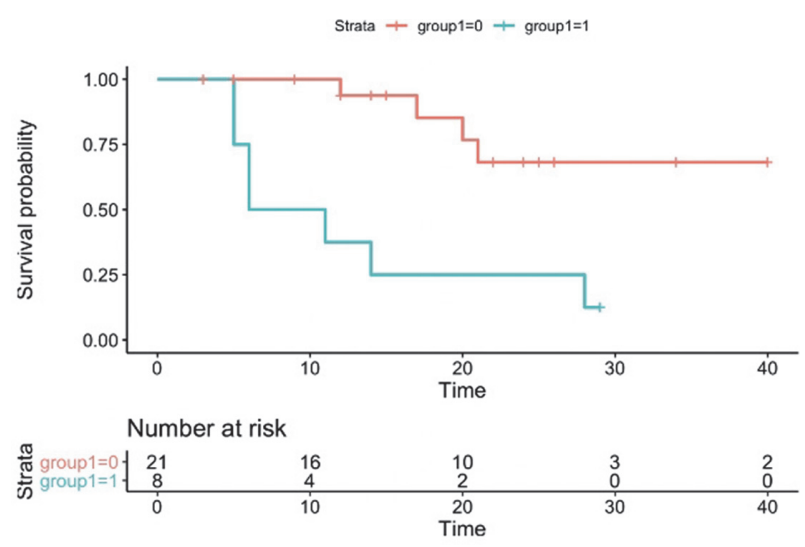

Abstract IDDF2021-ABS-0042 Figure 3

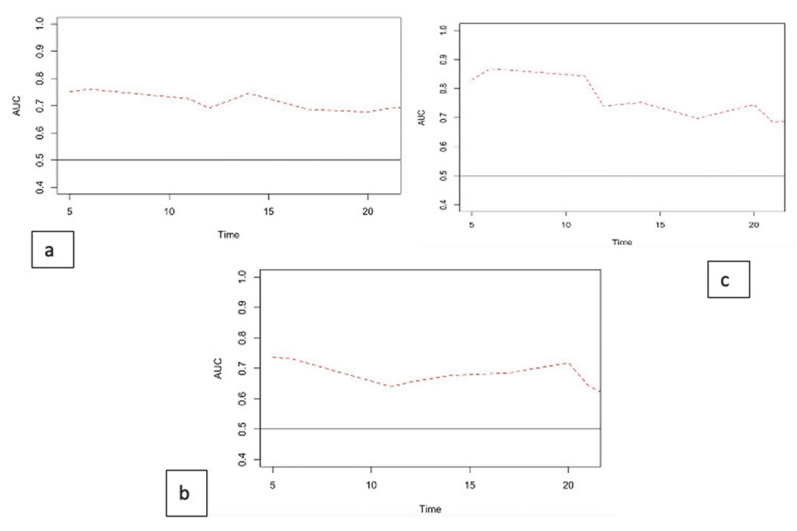

Abstract IDDF2021-ABS-0042 Figure 4
IDDF2021-ABS-0042 Figure 3. Overall survival of ART 2 groups,

IDDF2021-ABS-0042 Figure 4. Discrimatory ability of ABCR and ART).

Conclusions The ABCR and ART scores are simple and clinically relevant indexes, summing several variables endorsed in HCC. An ABCR $>3$ and ABCR $>1.5$ before the second TACE identify patient with dismal prognosis who may not benefit from further TACE

\section{IDDF2021-ABS-0045 EFFICACY OF MAC-2 BINDING PROTEIN GLYCOSYLATION ISOMER AS FIBROSIS PRESENCE AND SEVERITY BIOMARKER IN HEPATITIS: A SYSTEMATIC REVIEW}

${ }^{1}$ Rivaldo Heriyanto*, ${ }^{1}$ Jeannette Tandiono, ${ }^{1}$ Elizabeth Marcella, ${ }^{1}$ Billy Susanto, ${ }^{1}$ Sharon Chen, ${ }^{1}$ Charlotte Jonathan, ${ }^{2}$ Andree Kurniawan. ${ }^{1}$ Faculty of Medicine, Pelita Harapan University, Indonesia; ${ }^{2}$ Department of Internal Medicine Faculty of Medicine, Pelita Harapan University, Indonesia

\subsection{6/gutjnl-2021-IDDF.70}

Background In recent years, serum mac-2 binding Protein glycosylation isomer (M2BPGi) usefulness has been investigated in various liver diseases such as viral hepatitis and mortality in liver cirrhosis. However, its utilization in assessing the presence and severity of liver fibrosis in hepatitis patients remains unclear. Thus, this systematic review aims to investigate the efficacy of M2BPGi as a biomarker for assessing fibrosis presence and severity in hepatitis patients.

Methods We searched data from PMC, PubMed, Google Scholar, Science Direct, and Scopus, on March 12th, 2021, using a combination of keywords associated with the M2BPGi biomarker in correlation with its diagnostic efficacy in detecting fibrosis and its stages, and their variations or synonyms. Publications included are limited to English manuscripts that were published in the past 10 years. We excluded patients with liver diseases other than hepatitis. Diagnostic efficacy is measured by M2BPGi sensitivity, specificity, positive predictive values (PPV), negative predictive value (NPV), and area under the receiver operating characteristic (AUROC) curve. All studies are reviewed and evaluated by all 7 authors. The quality of each included study was assessed using the NewcastleOttawa Scale (NOS).

Results A total of 4 retrospective cohorts, 3 case-control, and 1 cross-sectional study were included consisting of 2116 patients. Based on NOS, all studies were good in quality. All studies showed that serum M2BPGi is a useful and reliable marker for non-invasive assessment and prediction of liver fibrosis in hepatitis patients. 4 studies also showed an association between serum M2BPGi level with fibrosis stages where higher M2BPGi level is correlated with more advanced stages of fibrosis.

Conclusions Recent studies demonstrated that serum M2BPGi level is proven to be associated with the presence of fibrosis, and increases with higher fibrosis stage, making it a promising biomarker in assessing the presence of fibrosis and its severity in hepatitis patients. Further studies should be warranted to investigate M2BPGi usefulness in assessing fibrosis in other liver diseases as well. 